\title{
Thrombophilia And Arterial Ischemic Stroke
}

Dr.A.A.Abrishamizadeh.M D,

Assistant Professor of Neurology, Fellowship of Interventional Neuroradiology Hormozgan University of Medical Sciences (Hums), Shahid Mohammadi Hospital Bandar-e-Abbas ,Iran.

*Corresponding Author: Email: aa_abrishami@yahoo.com

Ischemic stroke (IS) is a common cause of morbidity and mortality with significant socioeconomic impact especially when it affects young patients. Compared to the older adults, the incidence, risk factors, and etiology are distinctly different in younger IS. Hypercoagulable states are relatively more commonly detected in younger IS patients.

Thrombophilic states are disorders of hemostatic mechanisms that result in a predisposition to thrombosis .Thrombophilia is an established cause of venous thrombosis. Therefore, it is tempting to assume that these disorders might have a similar relationship with arterial thrombosis. Despite this fact that 1-4 \% of ischemic strokes are attributed to Thrombophillia, this alone rarely causes arterial occlusions .Even in individuals with a positive thrombophilia screen and arterial thrombosis, the former might not be the primary etiological factor.

Thrombophilic disorders can be broadly divided into inherited or acquired conditions. Inherited thrombophilic states include deficiencies of natural anticoagulants such as protein C, protein S, and antithrombin III (AT III) deficiency, polymorphisms causing resistance to activated protein $C$ (Factor $V$ Leiden mutation), and disturbance in the clotting balance (prothrombin gene 20210G/A variant). Of all the inherited thrombophilic disorders, Factor V Leiden mutation is perhaps the commonest cause. On the contrary, acquired thrombophilic disorders are more common and include conditions such as the antiphospholipid syndrome, associated with lupus anticoagulant and anticardiolipin antibodies.

The more useful and practical approach of ordering various diagnostic tests for the uncommon thrombophilic states tests should be determined by a detailed clinical history, physical examination, imaging studies and evaluating whether an underlying hypercoagulable state appears more likely.

The laboratory thrombophilia screening should be comprehensive and avoid missing the coexisting defect and It is important that a diagnostic search protocol includes tests for both inherited and acquired thrombophilic disorders.

Since the therapeutic approach (anticoagulation and thrombolytic therapy) determines the clinical outcomes, early diagnosis of the thrombophilic disorders plays an important role. Furthermore, the timing of test performance of some of the thrombophilic defects (like protein C, protein S, antithrombin III and fibrinogen levels) is often critical since these proteins can behave as acute phase reactants and erroneously elevated levels of these factors may be observed in patients with acute thrombotic events. On the other hand, the plasma levels of vitamin $\mathrm{K}$-dependent proteins (protein $\mathrm{C}$, protein $\mathrm{S}$ and APC resistance) may not be reliable in patients taking vitamin $\mathrm{K}$ antagonists. Therefore, it is suggested that plasma-based assays for these disorders should be repeated 3 to 6 months after the initial thrombotic episode to avoid false-positive results and avoid unnecessary prolonged anticoagulation therapy. The assays for these disorders are recommended after discontinuation of oral anticoagulant treatment or heparin for at least 2 weeks.

Key words: Thrombophillia, ischemic stroke 